RESEARCH

\title{
THE EFFECTS OF THIAZIDE AND THIAZIDE- LIKE DIURETICS ON THE RELATIONSHIP BETWEEN NORMAL SERUM URIC ACID LEVELS AND COGNITIVE FUNCTION
}

Turkish Journal of Geriatrics

DOI: 10.31086/tigeri.2021.250

2021; 24(4): 526-533

- Fatma Özge KAYHAN KOÇAK ${ }^{1}$

- Seval AY ${ }^{2}$

-Elif ERTUNA ${ }^{3}$

- Mehmet Zuhuri ARUN ${ }^{3}$

- Sevnaz ŞAHIN ${ }^{1}$

- Zeliha Fulden SARAÇ 1

- Selahattin Fehmi AKÇiÇEK ${ }^{1}$

CORRESPONDANCE

${ }^{1}$ Fatma Özge Kayhan KOÇAK

Ege University, Division of Geriatrics,

Department of Internal Medicine, izmir, Turkey

Phone: +905052845428

e-mail: drozgekayhankocak@gmail.com

Received: Sep 15, 2021

Accepted: Nov 20, 2021

${ }^{1}$ Ege University, Division of Geriatrics,

Department of Internal Medicine, izmir,

Turkey

${ }^{2}$ Istanbul Medeniyet University, Division of

Oncology, Department of Internal Medicine, istanbul, Turkey

${ }^{3}$ Ege University, Department of Clinical Pharmacy, Faculty of Pharmacy, izmir, Turkey

\section{Abstract}

Introduction: The objective of this study was to identify the possible effect of thiazide and thiazide-like diuretic use on the relationship between uric acid and cognitive function in geriatric patients with normal renal function.

Method: Serum uric acid levels and Mini-Mental State Examination scores of 238 patients (age $\geq 65$ years) treated in an outpatient geriatric clinic were evaluated in this observational, cross-sectional, retrospective study. The effect of thiazide diuretic use on Mini-Mental State Examination score was also assessed.

Results: Compared to patients that are not currently taking thiazide diuretics (non-thiazide group) serum uric acid was significantly higher in patients using thiazide and thiazide-like diuretics (thiazide group) $(5.27 \pm 0.12$ vs 6.09 $\pm 0.20 ; P<0.001)$. Although the serum uric acid levels were higher in the thiazide group the Mini-Mental State Examination scores were similar to the non-thiazide group. There was a strong positive correlation between serum uric acid levels and Mini-Mental State Examination score in both groups $(\mathrm{P}<$ 0.001). The Mini-Mental State Examination score was significantly lower in the lowest serum uric acid quartile in both groups $(P<0.001)$.

Conclusions: Low serum uric acid levels are associated with cognitive impairment; a slight but significant increase of serum uric acid due to thiazide diuretic use does not seem to enhance cognitive function in geriatric patients.

Keywords: Dementia; Cognitive Dysfunction; Geriatric Assessment; Uric Acid; Sodium Chloride Symporter Inhibitors; Aged. 


\section{INTRODUCTION}

Cognitive impairment has become one of the most urgent health problems in the world because of the rapid aging of its population (1). Reports from the World Health Organization indicate that cognitive impairment incidence will double every 20 years, especially in developing countries (2). Efforts to find new strategies to alleviate, slow, or delay the decline in cognitive function are ongoing.

Studies have revealed contradictory results on the correlation between serum uric acid (SUA) levels and cognitive functions. Du et al. discovered that higher SUA levels were associated with a lower risk of cognitive impairment and a decrease in dementia incidence (3). Contrary to this, in a population-based cohort study, Latourte et al. reported that the risk of dementia, especially vascular or mixed dementia, may be increased with high SUA levels in older adults (4), whereas Cao et al. followed 89 multiple system atrophy (MSA) patients for 2.5 years and found that low levels of SUA could be a predictor for cognitive decline in MSA patients (5).

Thiazide and thiazide-like diuretics are among the most commonly prescribed medications for the treatment of hypertension. Thiazide treatment is associated with a positive calcium balance and an increase in cortical bone mineral density (6), this feature might contribute to their selection as an antihypertensive agent in the elderly. Indeed, thiazide diuretics are frequently used in geriatric patients with hypertension (7). However, thiazide diuretics are known to cause SUA elevation (8). The correlation between the Mini Mental State Examination (MMSE) and SUA has been studied previously, but the results have been contradictory (9). It is not known whether thiazide-induced SUA elevation has beneficial or detrimental effects on cognitive function. Thus, we investigated the possible effect of thiazide and thiazide-like diuretic use on the relationship between SUA and the MMSE in geriatric patients with normal renal function.

\section{MATERIALS AND METHOD}

\section{Study population and data collection}

A total of 615 patients, aged 65 years or above and treated in the outpatient geriatric clinic of the Faculty of Medicine from November 2015 to December 2017, were evaluated in this observational, cross-sectional, retrospective study. The associated factors for predicting cognitive decline in relation to SUA are metabolic, nutritional, and genetic factors, as well as related diseases (diabetes mellitus, coronary heart disease) and lifestyle (10). Accordingly, patients were excluded from the study if they had acute or chronic renal failure (glomerular filtration rate $<60 \mathrm{ml} / \mathrm{min} / 1.73 \mathrm{~m}^{2}$ ); gout; hematological or oncological malignancy; previously confirmed dementia diagnosis; psychotic diseases (schizophrenia, affective disorder, refractory major depressive disorder, and similar diagnoses); New York Heart Association (NYHA) stage 3 or stage 4 heart failure; serious health conditions, such as sepsis, acute coronary syndrome, acute delirium, cerebrovascular event, gastrointestinal bleeding; hypothyroidism, hyperthyroidism or hyperparathyroidism; deafness; difficulty with verbal expression or mental retardation; hyponatremia or hypernatremia; hypokalemia or hyperkalemia (11); malnutrition (Mini Nutritional Assessment Short Form Scores < 7); and patients using drugs known to affect SUA levels (allopurinol, losartan, fenofibrate, acetylsalicylic acid, penicillin, levothyroxine, propylthiouracil, and alcohol abuse), with the exception of thiazide and thiazide-like diuretics. Patients treated with 12,5-25 mg/day hydrochlorothiazide or 1,25-5 mg/day indapamide for at least 6 months were included. Patients who died before giving informed consent were also excluded. After the exclusion of 377 patients due to the aforementioned reasons, 238 geriatric patients (158 females and 80 males) were included in this study.

Comprehensive geriatric assessment is routinely performed for each patient in our geriatric outpatient clinic. MMSE is one of the major components of comprehensive geriatric assessment. Patients' 
cognitive functions were assessed with the MMSE test. The MMSE includes 11 questions to evaluate five areas of cognitive function: orientation, memory, computation, language, and visual space (12). The highest score was 30 points, and cognitive functions were categorized as normal: 27-30, mild cognitive impairment: 24-26, moderate cognitive impairment: 18-23, and severe cognitive impairment: 0-17 (13). The MMSE score, blood biochemistry results, demographic and clinical data, were obtained retrospectively from patients' medical records.

\section{Biochemical assessments}

Laboratory tests were performed in the Department of Clinical Biochemistry Laboratory at the Faculty of Medicine, which is an accredited laboratory conforming to ISO 15189 standards.

\section{Ethics}

Authors complied with Good Clinical Practice standards throughout the study. This study is approved by the Ethics Committee for Clinical Research of Faculty of Medicine at Ege University (70198063-050.06.04) and is conducted according to the World Medical Association Declaration of Helsinki. Informed consent was obtained from all individual participants included in the study.

\section{Statistical analysis}

The database was constructed using Excel. Continuous variables were expressed as means \pm SEM. Patients were divided into two subgroups: patients taking thiazide and thiazide-like diuretics (the thiazide group) and patients that are not currently taking thiazide diuretics (the non-thiazide group). The two groups were compared using the t-test. Nominal values (such as sex) in the two groups were compared using the Chi-square test. The correlation between the MMSE score and SUA levels was determined using Pearson correlation coefficient.
Analysis of variance, followed by Tukey, were used for comparing SUA quartiles. All statistical tests were performed using $\mathrm{R}$ software (ver. 3.4.3). $P \leq$ 0.05 was considered statistically significant.

\section{RESULTS}

\section{Demographic characteristics}

This study included 238 geriatric patients (158 females and 80 males). Hypertension was found in 164 (57.1\%) patients, 84 (51.2\%) of the patients with hypertension were taking thiazide diuretics. There was no statistically significant difference in age between the non-thiazide group and the thiazide group. There were no differences in demographic characteristics between these groups (Table 1).

\section{Effect of thiazide diuretics on laboratory values}

Parenchymal disease of the liver, renal disease, and serum sodium and potassium levels are known to affect uric acid metabolism (14). To determine the possible confounding effect of these factors on SUA levels, total and direct bilirubin, alanine aminotransferase (ALT), aspartate aminotransferase (AST), serum creatinine, serum sodium, and potassium values were compared. There was no significant difference in these parameters between the non-thiazide group and the thiazide group (Table 1). Normal SUA level ranges from 4,0 to $8,5 \mathrm{mg} / \mathrm{dL}$ for adult males and 2,7 to 7,3 mg/dL for adult females, and a slight increase in these values may occur in the elderly. The SUA levels of all of the patients included in this study were within the reference range. Compared to the non-thiazide group, SUA levels were significantly higher in the thiazide group $(5,265 \pm 0,1214$ vs $6,094 \pm 0,1977 \mathrm{mg} / \mathrm{dL}, \mathrm{P}<0.001$; Table 1). Similarly, serum urea levels were also significantly higher in the thiazide group $(37,27 \pm 0,8607$ vs 42,38 $\pm 1,444 \mathrm{mg} / \mathrm{dL}, \mathrm{P}<0.05$; Table 1).

Serum uric acid levels and their correlation with the MMSE score 
Table 1. Demographic characteristics and laboratory values of patients.

\begin{tabular}{|c|c|c|c|c|}
\hline & & $\begin{array}{c}\text { Non-thiazide group } \\
\qquad(n=166)\end{array}$ & $\begin{array}{l}\text { Thiazide Group } \\
(n=72)\end{array}$ & $\boldsymbol{P}$ \\
\hline \multirow[t]{2}{*}{ Sex } & Female & 108 & 50 & \multirow[b]{2}{*}{0.5526} \\
\hline & Male & 58 & 22 & \\
\hline \multicolumn{2}{|l|}{ Age } & $77.69 \pm 0.59$ & $75.81 \pm 0.79$ & 0.0708 \\
\hline \multicolumn{2}{|c|}{ MMSE score } & $24.21 \pm 0.45$ & $25.18 \pm 0.69$ & 0.2351 \\
\hline \multicolumn{2}{|c|}{ Serum Uric Acid (mg/dL) } & $5.27 \pm 0.12$ & $6.09 \pm 0.20$ & $0.0003 * \star \star$ \\
\hline \multicolumn{2}{|c|}{ Urea $(\mathrm{mg} / \mathrm{dL})$} & $37.27 \pm 0.86$ & $42.38 \pm 1.44$ & $0.0018 * * *$ \\
\hline \multicolumn{2}{|c|}{ Creatinine (mg/dL) } & $0.85 \pm 0.03$ & $0.85 \pm 0.02$ & 0.9785 \\
\hline \multicolumn{2}{|c|}{ Sodium (mEq/L) } & $140.30 \pm 0.25$ & $140 \pm 0.40$ & 0.5970 \\
\hline \multicolumn{2}{|c|}{ Potassium (mEq/L) } & $4.52 \pm 0.04$ & $4.52 \pm 0.05$ & 0.9451 \\
\hline \multicolumn{2}{|c|}{ Total bilirubin (mg/dL) } & $0.60 \pm 0.07$ & $0.49 \pm 0.02$ & 0.2816 \\
\hline \multicolumn{2}{|c|}{ Direct bilirubin (mg/dL) } & $0.23 \pm 0.02$ & $0.38 \pm 0.18$ & 0.2293 \\
\hline \multicolumn{2}{|c|}{$A L T(U / L)$} & $15.48 \pm 0.58$ & $14.51 \pm 0.59$ & 0.3105 \\
\hline \multicolumn{2}{|c|}{$A S T(U / L)$} & $19.13 \pm 0.54$ & $17.27 \pm 0.50$ & 0.0748 \\
\hline
\end{tabular}

** $\mathrm{P} \leq 0.005,{ }^{* \star *} \mathrm{P} \leq 0.001 ;$ Student's t-test.

As SUA levels were found to be higher in the thiazide group, the correlation between the MMSE score and SUA levels was computed separately. There was a strong positive correlation between SUA levels and the MMSE score in both groups $(P<0.001$; Figure 1). However, there was no significant difference in the MMSE score between the thiazide group and the non-thiazide group ( $P=0.2351)$.

To compare the MMSE scores of patients that had different SUA values, we evaluated the MMSE scores according to SUA quartiles. ANOVA and post-hoc analysis showed that the mean MMSE score of the $<25^{\text {th }}$ SUA quartile was significantly lower than other quartiles in both groups (Figure 2).

\section{DISCUSSION}

In this observational, cross-sectional, retrospective study, we evaluated the association of SUA levels and the cognitive function of geriatric patients in the outpatient geriatric clinic. Patients with diseases affecting uric acid metabolism and previously confirmed dementia diagnosis were not included in the study. Our results showed that the SUA levels were higher in the thiazide group. In elderly patients with normal renal function, the SUA levels were positively correlated with cognitive function; however, there was no difference in cognitive function between patients who were using thiazide diuretics and those who were not.

Our findings regarding the relationship between SUA and cognitive function are consistent with the results of other studies. In a prospective population-based cohort study, higher SUA levels were associated with a decreased risk of dementia (15). Molshatzki et al. followed SUA levels and cognitive function in patients with pre-existing cardiovascular disease for 10 years and reported that low SUA levels are associated with poorer cognitive function (16). Likewise, low SUA levels are related to poor 
Figure 1. Correlation between serum uric acid and MMSE Score by Pearson test.
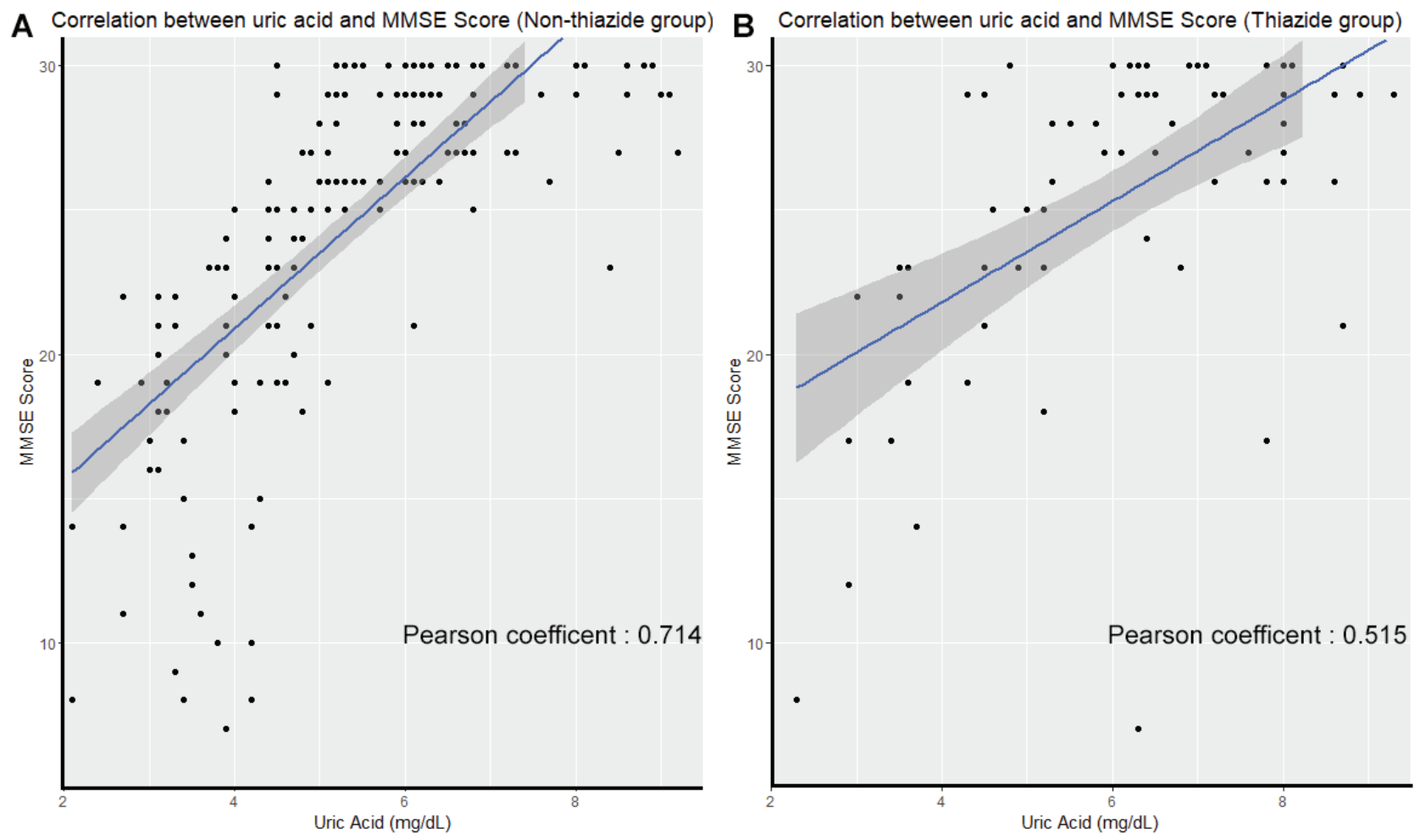

A. The non-thiazide group $(n=166), B$. The thiazide group $(n=72)$.

cognitive scores in our study; the mean MMSE score of the $<25^{\text {th }}$ SUA quartile was significantly lower than those of the other quartiles (Figure 2). In patients with normal renal function, a higher SUA level was associated with a higher MMSE score and a lower risk of cognitive impairment. This may be due to the antioxidant effect of SUA. The pathophysiology of cognitive impairment is associated with an increase in oxidative stress factors, such as reactive oxygen species (ROS), and a decrease in antioxidant levels, such as nitric oxide (NO) (17). SUA has both prooxidative effects (inhibition of endothelial NO synthase) and antioxidative effects (inhibition of nitrite-mediated nitrification and iron-dependent oxidative damage on the vascular system) and could have free-radical scavenging activity in brain tissues (18).

On the other hand, Suzuki et al. showed that high SUA levels can lead to a microinflammatory state and produce oxidative stress, resulting in microvascular disease, increased cerebral ischemia, and hypoxia, increasing the risk of cognitive impairment (19). There are some contradictory data about SUA and cognitive impairment: one study with 247 chronic renal failure (CRF) patients has shown that increased SUA levels may be associated with worse cognitive function (20). The study was designed with CRF patients whose SUA levels were between $13.4 \mathrm{mg} / \mathrm{dL}$ and $17.7 \mathrm{mg} / \mathrm{dL}$. SUA has paradoxical biological effects; it acts as an antioxidant in an ex- 
Figure 2. Mean MMSE scores according to serum uric acid quartiles.

A

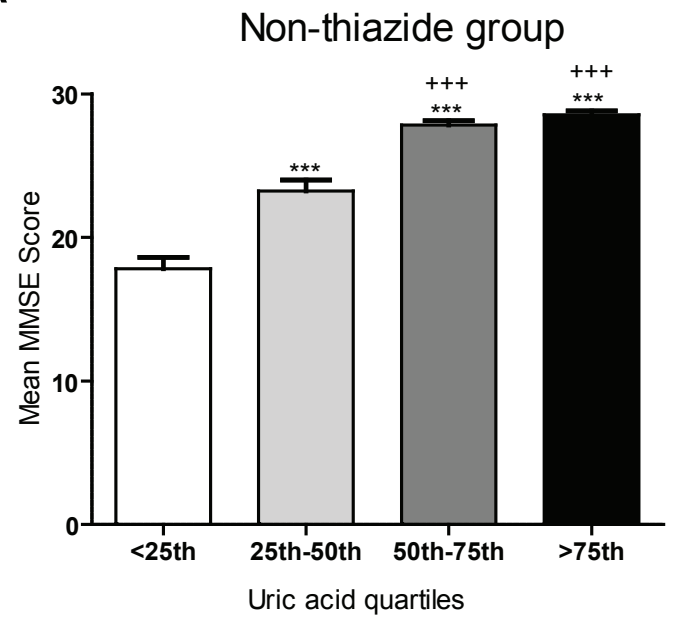

B

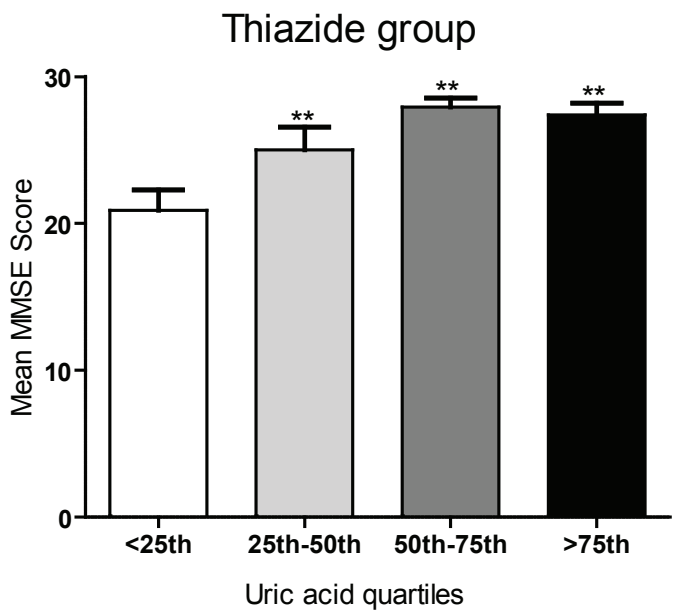

A) The non-thiazide group: $<25^{\text {th }}$ quartile $(n=43)<4 \mathrm{mg} / \mathrm{dL} ; 25^{\text {th }}-50^{\text {th }}$ quartile $(n=43) 4-5.2 \mathrm{mg} / \mathrm{dL} ; 50^{\text {th }}-75^{\text {th }}$ quartile $(\mathrm{n}=40) 5.2-6.2 \mathrm{mg} /$ $\mathrm{dL} ;>75^{\text {th }}$ quartile $\left.(\mathrm{n}=40)>6.2 \mathrm{mg} / \mathrm{dL} ; \mathrm{B}\right)$ The thiazide group $<25^{\text {th }}$ quartile $(\mathrm{n}=18):<4.97 \mathrm{mg} / \mathrm{dL} ; 25^{\text {th }}-50^{\text {th }}$ quartile $(\mathrm{n}=21) 4.97-6.3 \mathrm{mg} /$ $\mathrm{dL} ; 50^{\text {th }}-75^{\text {th }}$ quartile $(n=15) 6.3-7.23 \mathrm{mg} / \mathrm{dL} ;>75^{\text {th }}$ quartile $(n=18)>7.23 \mathrm{mg} / \mathrm{dL}$. ANOVA followed by Tukey. ** $P \leq 0.005, * \star * ~ P \leq 0.001 \mathrm{vs}<$ $25^{\text {th }}$ quartile; ${ }^{+++} P \leq 0.001$ vs $25^{\text {th }}-50^{\text {th }}$ quartile.

tracellular environment and a prooxidant in an intracellular environment. UA in the kidney triggers oxidative stress (21). Also, CRF is an independent risk factor for cognitive impairment (22). Thus, the conflicting results of Afsar et al. about the negative relationship between high SUA and cognitive function might be due to the presence of CRF and pathological SUA levels (23).

Thiazide diuretics are frequently used in geriatric patients with hypertension (24); however, there are limited studies investigating the correlation between SUA and cognitive impairment in patients using these drugs. Several clinical studies have reported that thiazide diuretics reduce the risk of dementia. In our study, as expected, we found that SUA levels were higher in patients using thiazide diuretics. There was a strong positive correlation between SUA levels and the MMSE scores in both groups. To compare the MMSE scores of patients that have different serum UA values, we evaluated
MMSE scores according to quartiles of SUA, and the mean MMSE score of the $>50^{\text {th }}-75^{\text {th }}$ UA quartile was significantly higher than others (Figure 2). Although the SUA level was higher in thiazide diuretic users, the correlation between the MMSE scores and SUA levels was similar in both groups. Koçyiğit et al. demonstrated that 26-week thiazide therapy was not related to any changes in the MMSE scores in older hypertensive adults. However, the SUA was not elevated by thiazides in this study (25). A 26week follow-up period may not be enough to cause a significant increase in SUA, resulting in no alteration in the MMSE scores; therefore, a direct comparison with our results would be futile.

This study has several limitations. First, it was conducted retrospectively; a randomized controlled trial design would have been more suitable to assess the causality of the relationship between SUA and cognitive outcomes more thoroughly. Although the MMSE is still the most commonly 
used tool for cognitive screening, other neuropsychiatric scales and neuroimaging tests could have been used to support our findings. The etiology of cognitive impairment could not be identified for all patients in our study, and the inclusion of different dementia types might contribute to controversial results among studies. Only patients with normal SUA levels were included in this study, thus the relationship between cognitive functions and SUA was only evaluated within normal SUA limits. The effect of high SUA levels was not investigated. This study

\section{REFERENCES}

1. Prince M, Bryce R, Albanese E, Wimo A, Ribeiro W, Ferri CP. The global prevalence of dementia: A systematic review and metaanalysis. Alzheimer's Dement 2013;9(1):63-75.e2. (PMID: 23305823)

2. Wortmann M. Dementia: a global health priority highlights from an ADI and World Health Organization report. Alzheimers Res Ther 2012;4(5):40. (PMID: 22995353)

3. Du N, Xu D, Hou X, et al. Inverse Association Between Serum Uric Acid Levels and Alzheimer's Disease Risk. Mol Neurobiol 2016; 53(4): 2594-2599. (PMID: 26084440)

4. Latourte A, Soumaré A, Bardin T, Perez-Ruiz F, Debette $S$, Richette $P$. Uric acid and incident dementia over 12 years of follow-up: a population-based cohort study. Ann Rheum Dis 2018;77(3):328-335. (PMID: 28754803)

5. Cao B, Wei Q-O, Ou R, Yang J, Shang H-F. Association of serum uric acid level with cognitive function among patients with multiple system atrophy. J Neurol Sci 2015;359(1-2): 363-366. (PMID: 26671143)

6. Dvorak MM, De Joussineau C, Carter DH, et al. Thiazide Diuretics Directly Induce Osteoblast Differentiation and Mineralized Nodule Formation by Interacting with a Sodium Chloride Co-Transporter in Bone. J Am Soc Nephrol 2007;18(9):2509-2516. (PMID: 17656470)

7. Arıcı M, Birdane A, Güler K, et al. Turkish Hypertension Consensus Report. Archives of the Turkish Society of Cardiology 2015;43(4):402-409. (PMID: 26142798) (in Turkish) included both normotensive and hypertensive patients. Therefore, the groups were not matched for hypertension, which increases the risk of dementia.

In summary, in geriatric patients with normal renal function, there is a positive correlation between SUA levels and MMSE scores, indicating that SUA may play a protective role against cognitive impairment. However, the SUA-elevating effect of thiazide diuretics does not seem to enhance the preservation of cognitive function.

8. Raja R, Kavita F, Amreek F, Shah A, Sayeed KA, Sehar A. Hyperuricemia Associated with Thiazide Diuretics in Hypertensive Adults. Cureus. 2019;11(8):e5457. (PMID: 31641556)

9. Wang F, Zhao M, Han Z, et al. Long-Term Subclinical Hyperglycemia and Hypoglycemia as Independent Risk Factors for Mild Cognitive Impairment in Elderly People. Tohoku J Exp Med 2017;242(2):121-128. (PMID: 28592718)

10. Niskanen LK, Laaksonen DE, Nyyssönen K, et al. Uric Acid Level as a Risk Factor for Cardiovascular and All-Cause Mortality in Middle-aged Men. Arch Intern Med 2004;164(14): 1546-1551. (PMID: 15277287)

11. Folstein MF, Folstein SE, McHugh PR. "Mini-mental state" A practical method for grading the cognitive state of patients for the clinician. J Psychiatr Res 1975;12(3):189-198. (PMID: 1202204)

12. Keskinoglu P, Ucku R, Yener G, Yaka E, Kurt P, Tunca Z. Reliability and validity of revised Turkish version of Mini Mental State Examination (rMMSE-T) in community-dwelling educated and uneducated elderly. Int J Geriatr Psychiatry 2009;24(11):1242-1250. (PMID: 19337986)

13. Molloy DW, Standish TI. A guide to the standardized Mini-Mental State Examination. Int psychogeriatrics 1997;9(1):50-87. (PMID: 9447431)

14. Darmawan G, Hamijoyo L, Hasan I. Association between Serum Uric Acid and Non-Alcoholic Fatty Liver Disease: A Meta-Analysis. Acta Med Indones 2017;49(2):136-147. (PMID: 28790228)

15. Li F, Guo H, Zou J, et al. The Association of Urinary Sodium and Potassium with Renal Uric Acid Excre- 
tion in Patients with Chronic Kidney Disease. Kidney Blood Press Res 2018;43(4):1310-1321. (PMID: 30099444)

16. Xiu S, Zheng Z, Guan S, Zhang J, Ma J, Chan P. Serum uric acid and impaired cognitive function in community-dwelling elderly in Beijing. Neurosci Lett 2017;637(10):182-187. (PMID: 27890742)

17. Molshatzki N, Weinstein G, Streifler JY, Goldbourt U, Tanne D. Serum Uric Acid and Subsequent Cognitive Performance in Patients with Pre-Existing Cardiovascular Disease. PLoS One 2015;10(3):e0120862. (PMID: 25794156)

18. Ansari MA, Scheff SW. Oxidative Stress in the Progression of Alzheimer Disease in the Frontal Cortex. J Neuropathol Exp Neurol 2010;69(2):155-167. (PMID: 20084018)

19. Kim TS, Pae CU, Yoon SJ, et al. Decreased plasma antioxidants in patients with Alzheimer's disease. Int J Geriatr Psychiatry 2006;21(4):344-348. (PMID: 16534775)

20. Davies KJ, Sevanian A, Muakkassah-Kelly SF, Hochstein P. Uric acid-iron ion complexes. A new aspect of the antioxidant functions of uric acid. Biochem J 1986;235(3):747-754. (PMID: 3753442)
21. Suzuki K, Koide D, Fujii K, Yamazaki T, Tsuji S, Iwata A. Elevated Serum Uric Acid Levels Are Related to Cognitive Deterioration in an Elderly Japanese Population. Dement Geriatr Cogn Dis Extra 2016;6(3):580-588. (PMID: 28203247)

22. Afsar B, Elsurer R, Covic A, Johnson RJ, Kanbay M. Relationship between Uric Acid and Subtle Cognitive Dysfunction in Chronic Kidney Disease. Am J Nephrol 2011;34(1):49-54. (PMID: 21659739)

23. Roumeliotis S, Roumeliotis A, Dounousi E, Eleftheriadis T, Liakopoukos V. Dietary Antioxidant Supplements and Uric Acid in Chronic Kidney Disease: A Review. Nutrients; 2019;11(8):1911. (PMID: 31443225)

24. Viggiano D, Wagner CA, Blankestijn PJ, et al. Mild cognitive impairment and kidney disease: clinical aspects. Nephrol Dial Transplant 2020;35(1):10-17. (PMID: 31071220)

25. Kocyigit SE, Soysal P, Ates Bulut E, Dokuzlar O, Isık AT. Thiazide therapy is not related to any changes in cognitive function in older hypertensive patients with or without dementia: a 26-week follow-up study. Psychogeriatrics 2019;19(1):16-22. (PMID: 30058745) 